\title{
Should We Walk or Take a Car for Minimum Greenhouse Gas Emissions?
}

\author{
Rafig Babayev, Bengt Johansson
}

King Abdullah University of Science and Technology (KAUST)

\begin{abstract}
This paper compares the greenhouse gas (GHG) emissions attributed to driving a popular production vehicle powered by an internal combustion engine (ICE), as well as a hybrid electric vehicle (HEV), with GHG emissions associated with walking, running and bicycling. The purpose of this study is to offer a different perspective on the problem of global warming due to anthropogenic causes, specifically on transportation and eating patterns. In order to accurately estimate emissions, a full life cycle of food has been considered coupled with energy expenditures of the aforementioned activities obtained from several different sources and averaged for more reliable results. The GHG emissions were calculated for Sweden, the UK, and the US. Depending on the availability of certain data, the methodology for different countries was altered slightly. The question whether walking, running or taking a bicycle is better for the environment than driving a car cannot be answered uniquely. This study demonstrates that the answer depends on several factors, such as diet composition, the number of people commuting, vehicle powertrain, as well as the country analyzed. The conclusion is that if one has an eco-friendly diet and travels alone the preferred modes of transport would be bicycling, walking and running, the cleanest of which by far is bicycling. However, if the diet has a higher $\mathrm{CO} 2$ footprint, as in the case of diets containing a large amount of meat and/or imported products, then the preference shifts towards cars, among which the most environmentally friendly are hybrid electric vehicles. The same conclusion applies to the cases where the number of people commuting together exceeds two-three persons.
\end{abstract}

\section{Introduction}

Cars powered by internal combustion engines have long been associated with greenhouse gas emissions. It has been a subject undergoing intense study and a multitude of articles have been published encouraging the governments to ban ICE powered vehicles in favor of other means of transportation, such as battery-electric vehicles (BEVs), bicycles, or even walking/running [1-3]. However, the issue of environmental degradation due to anthropogenic causes is much more profound and has to be studied more thoroughly. In particular, the life-cycle GHG emissions of food needed to replenish calories burned while walking, running, bicycling and driving a car are rarely taken into account. This creates the misconception of the environmental consequences of different activities. The most typical assumption made when studying the benefits of human-powered modes of transportation is that they are almost or completely emission-free [4-6]. The benefits used to be described mainly in terms of the local emissions, such as carbon monoxide (CO), nitrogen

Page 1 of 15 oxides (NOx), and volatile organic compounds (VOCs). With the developments in engines and after-treatment system technologies in the automotive industry, the focus was shifted towards GHG emissions, the dominant of which is carbon dioxide (CO2). This paper is aimed to put the GHG emissions attributed to the motorized and active transport into perspective, by comparing the amount of $\mathrm{CO} 2 \mathrm{e}$ emitted per kilometer traveled walking, running, bicycling and driving a car. This is achieved by taking into account the energy expended by the human body performing the aforementioned activities, estimating the amount of food in the form of caloric content of different diets that need to be consumed in order to compensate for the energy loss associated with these activities, and the amount of GHG emitted in the production of the foods comprising the diets. The latter is calculated based on the Life Cycle Assessment (LCA) of the food. The caloric content of food in this study is taken from the USDA National Nutrient Database [7], which uses the Atwater Specific Factor System. This system already includes all the losses and inefficiencies associated with transformation of the total energy stored in the food into the form of energy used by human body to generate mechanical work. The details are given in the "Food Energy Content" section.

The analysis described above enables the estimation of the amount of GHG (CO2e) emitted per distance traveled for different means of transportation, which allows a direct comparison of the emissions attributed to walking, running and bicycling with the emissions associated with driving a car, ICE powered or hybrid. The car emissions, in turn, are calculated based on the Well-to-Wheels (WTW) analysis, which can be assumed equivalent to LCA of foods in the context of the current paper. This assumption is justified later in the introduction.

The following three countries are analyzed separately: (1) Sweden, (2) UK, (3) USA. The reason lies mainly in the drastically different GHG emissions associated with food production in different countries. The countries are selected based on the availability and reliability of the data needed for the analysis. It was also decided to develop different procedures of estimating GHG emissions of walking, bicycling, running and driving a car depending on the country it is applicable to. The reason for that was the scarcity of certain data for individual countries. On the other hand, this decision helped diversify the analysis. Methods used in this study are described in the "Methodology" section.

In order to make a valid comparison between the emissions of human-powered transportation methods and the emissions concomitant with driving a car, the WTW emission values are taken for a vehicle with conventional powertrain (Opel Astra) and a hybrid 
vehicle (Toyota Prius). WTW analysis evaluates: (1) GHG emissions, (2) Energy efficiency, (3) Industrial costs of different powertrains and fuels for automotive vehicles. It can further be split into two categories: (a) Well-to-Tank (WTT) analysis, and (b) Tank-toWheels (TTW) analysis.

(a) The WTT analysis estimates the energy required and GHGs emitted to deliver fuel to the on-board fuel tank of a vehicle. It covers all processes involved, including extracting, capturing or growing the primary fuel, its transportation, refinement into the road fuel, distribution and refueling of vehicles. Production costs and fuel availability are taken into account as well.

(b) The TTW analysis estimates the energy required and GHGs emitted during the transformation of the fuel energy into mechanical energy in a vehicle in accordance with a reference driving cycle, which is the New European Driving Cycle (NEDC) [8] in this study.

Particular emphasis should be put on the fact that WTW analysis is not the same as LCA of a vehicle, since it neglects the energy expenditure and GHG emissions associated with production of vehicles as well as construction of the factories and facilities involved, but focuses mainly on automotive fuels and powertrains. In the context of this study the LCA of foods and WTW analysis of vehicles are directly compared based on the fact that food can be viewed as a "fuel" for a "powertrain" that is human body. The final WTW GHG emissions obtained from this analysis are $151 \mathrm{gCO} 2 \mathrm{e} / \mathrm{km}$ for a vehicle with a conventional powertrain (Opel Astra in this case) [9], 16\% of which come from WTT emissions. For comparison, Toyota Prius is estimated to have WTW GHG emissions about 95 $\mathrm{gCO} 2 \mathrm{e} / \mathrm{km}$, which is $37 \%$ lower than that of Opel Astra [10]. WTT emissions of Prius still constitute about $16 \%$ of the total WTW emissions. The calculations are based on an adapted procedure suggested by the European Commission-Joint Research Center [1112]. This procedure is presented in Appendix A. It should also be noted that the dietary $\mathrm{CO} 2$ emissions of the driver and the passengers were added to the WTW emissions of the vehicle itself.

To accurately estimate the GHG emissions of an automobile with passengers the mass of the passengers is also considered. As the number of passengers increases the vehicle becomes heavier leading to a higher fuel consumption, which, in turn, results in higher $\mathrm{CO} 2$ emissions. Relation between fuel consumption and the mass added to the vehicle was studied by Pagerit et al. at Argonne National Laboratory [13]. According to this study $10 \%$ increase in vehicle mass leads to $4.1 \%$ increase in fuel consumption (on a $\mathrm{L} / 100 \mathrm{~km}$ basis), while $20 \%$ increase in vehicle mass leads to $8.2 \%$ increase in fuel consumption. The values were given for a midsize vehicle with conventional powertrain under the UDDS cycle (the driving cycle effect was minimal). In this study, the increase in the percentage of vehicle mass with every passenger added is calculated assuming the vehicle mass with a driver is $1544 \mathrm{~kg}$ and the mass of each passenger is $71 \mathrm{~kg}$. The number of passengers is varied from 1 to 4 . Then, the percentage increase in fuel consumption is obtained based on the values suggested by Pagerit et al. using linear interpolation, since the relation is approximately linear.

Another aspect that influences the GHG emissions of a vehicle is the cold start. The vehicle fuel consumption, and hence, GHG emissions are higher when the engine is cold, due to higher friction losses and changes in equivalence ratio. The NEDC procedure begins with a cold start, hence, in the beginning of the test cycle when the engine is still warming up, the emissions are higher than usual. This introduces a non-linearity to the results. This effect was assumed negligible in this study. The NEDC covers approximately $11 \mathrm{~km}$, consequently, the emission values presented are expected to be slightly

Page 2 of 15 underestimated if one drives a distance shorter than that. The opposite also applies.

In this study, the carbon dioxide emissions attributed to breathing are also evaluated. The methods and results are presented in Appendix B. The respiratory carbon dioxide is proven to be significant, adding 10$30 \%$ to the total emissions of a person commuting. However, it was decided not to add it to the total GHG emissions when making the comparison. The reason for that is the fact that people are a part of the natural carbon cycle, and the $\mathrm{CO} 2$ we expel will later be used by plants to produce oxygen. This is, however, not the case for the industrialized food production, which causes liberation of far more carbon than the environment can effectively process.

Finally, given all the background information needed for comparison, the details of the methodology used to estimate the GHG emissions attributed to walking, running and bicycling are described in the subsequent section.

\section{Methodology}

The methodology used in the current study to estimate the GHG emissions of different means of transportation is summarized in Figure 1. Each step of the method is explained in the subsequent sections, starting from the "Energy Expenditure", followed by the "Food Energy Content", and finalized with the "Food Production GHGs" section. The "Energy Expenditure" section is applicable to all three countries, while the "Food Energy Content" section reports values for Sweden in $\mathrm{kWh} / \mathrm{kg}_{\text {food, }}$, and for the US in $\mathrm{kWh} /$ day, as per the methodology described in Figure 1. The "Food Production GHGs" section contains the values for Sweden in $\mathrm{gCO} 2 \mathrm{e} / \mathrm{kg}_{\text {food, }}$, the US in $\mathrm{gCO} 2 \mathrm{e} / \mathrm{day}$, and the UK in $\mathrm{gCO} 2 \mathrm{e} / \mathrm{kWh}$.

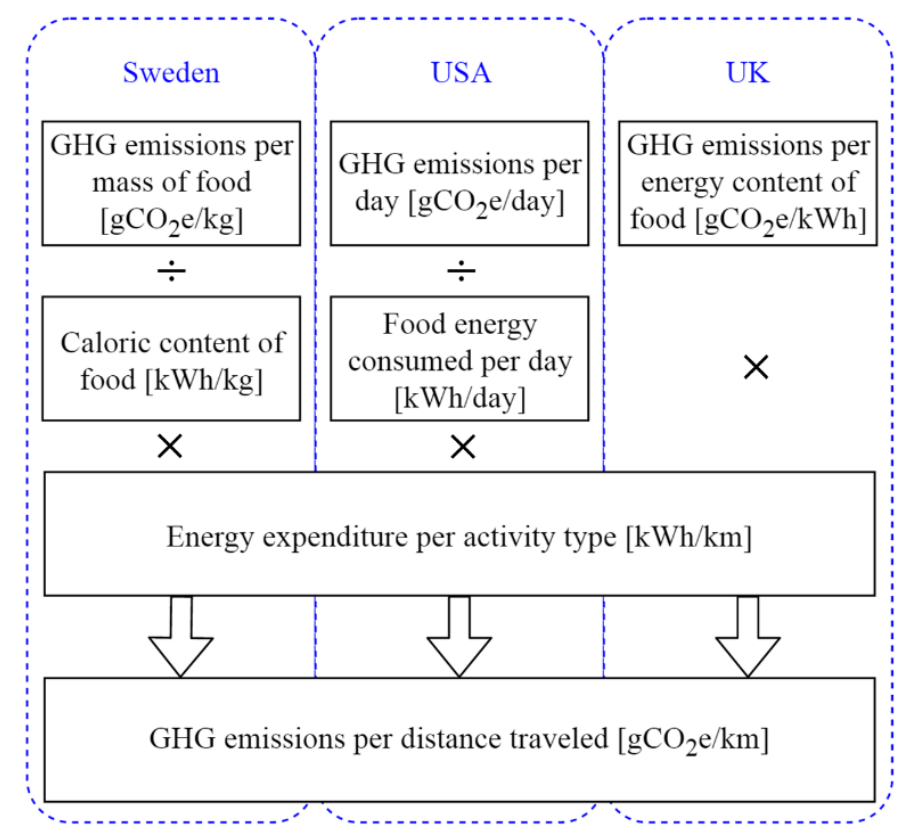

Figure 1. The flowchart of estimating GHG emissions of different transportation modes. 


\section{Energy Expenditure}

The first step in the calculation of GHG emissions of humans during different activities is the identification of the energy expenditure of the activities. The reported data varied substantially depending on the data source. To ensure the accuracy of the study, several different sources are analyzed to obtain the mean values and the Standard Error (SE). The data points are presented in Figure 2 as diamonds. Mean and SE are illustrated in the form of horizontal lines and error bars, respectively.

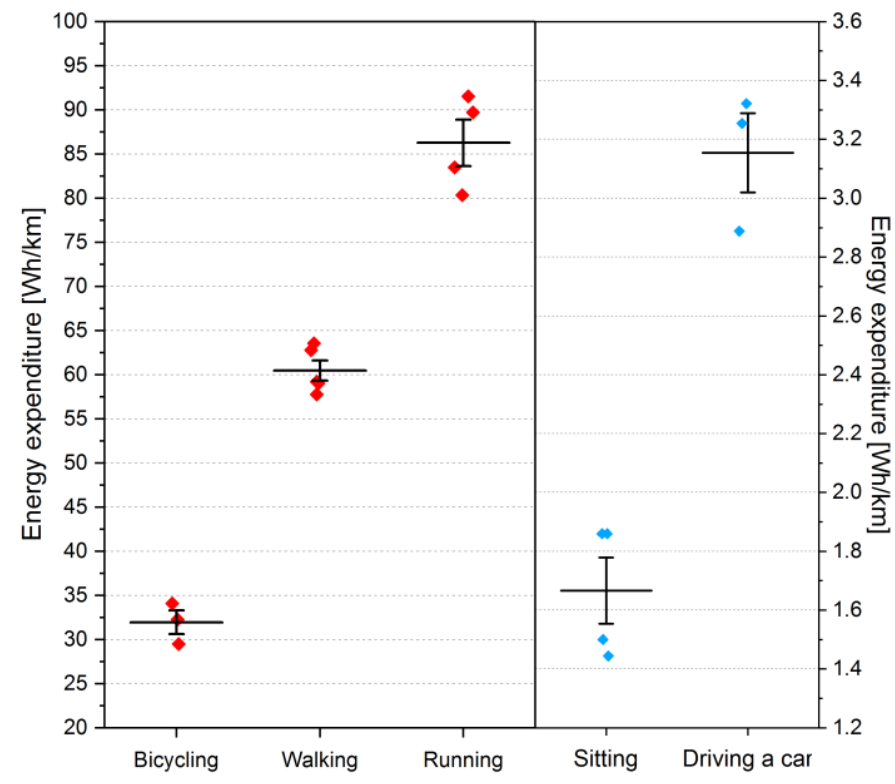

Figure 2. Energy expenditure of different activities in $\mathrm{Wh} / \mathrm{km}$. The horizontal lines indicate mean values, and the error bars indicate standard error. The diamonds represent data points $[14,15,16,17,18,19,20,21,22]$.

The values summarized in this study were reported by several authors and were estimated using different techniques. Some are based on recording the time spent in each of the various activities and measuring their energy cost by indirect calorimetry [14]. Others are based on the measurement of peak aerobic power ( $\mathrm{VO}_{2}$ peak) and converting it to kilojoules assuming that $1 \mathrm{~mL}$ of oxygen consumed produces 20.1 joules of energy $[15,17,23,24,25,26]$. There is a multitude of models used to predict $\mathrm{VO}_{2}$ peak. Some of them are listed in Appendix C. $\mathrm{VO}_{2}$ peak can then be multiplied by the amount of energy produced per ml of oxygen consumed by the human body and the body weight to get the energy expenditure per unit time. For this study, these values were divided by the speed to obtain the energy consumption per unit distance.

Another way of estimating the energy consumption of humans during different activities extensively used in this study is the method developed by the University of Colorado Hospital [16]. The prediction is made by using the following equation:

$$
\text { EnergyCons. }\left[\frac{k c a l}{\min }\right]=0.0175 \times M E T \times W
$$

Metabolic equivalent represents the amount of energy expended and oxygen consumed while performing a specific activity. One MET is equivalent to the amount of energy expended while sitting still. During exercise, the MET level increases as the result of higher energy and oxygen demand of the working muscles. METs are retrieved from the charts by different sources, including Jette et al. [18] as well as the University of Colorado Hospital [16].

The human energy expenditure is usually recorded at steady state, hence, the speed variations during walking, running, and bicycling are not taken into account. The average walking, running, and bicycling speeds are assumed to be $4.8 \mathrm{~km} / \mathrm{h}, 9.7 \mathrm{~km} / \mathrm{h}$, and 16.0 $\mathrm{km} / \mathrm{h}$, respectively. These are the average values taken from the sources of the energy expenditure data. The car speed is assumed to be $60 \mathrm{~km} / \mathrm{h}$. As seen from Figure 2, running is the most energyintensive activity out of all physical activities considered. Next comes walking, then bicycling. It can also be observed that the least energyintensive activities considered are driving a car and sitting still (as a passenger), accounting for only $3.6 \%$ and $2 \%$ of the energy consumption of running, respectively.

\section{Food Energy Content}

The analysis of food energy content was done for 3 countries and several different diets. Details are described below:

1. Swedish diets:
(a) Beef-only diet
(b) Diet A
(c) Diet $B$
(d) Diet C

2. The US standard diet

3. The UK diets:
(a) Beef-only diet
(b) Vegetarian diet
(c) Medium-meat diet
(d) High-meat diet

It should be noted that, even though the assumption of a regular diet consisting only of beef is not reasonable, the beef-only diet is still considered in this article to account for all occasions when a person opts to replenish the calories lost commuting by eating meat. In addition, analyzing this diet helps to show the crucial role of meat in the total emissions of regular diets.

\section{Sweden}

Table 1 lists the constituents of the three Swedish diets and their caloric ratings.

where $\mathrm{W}$ - body mass $[\mathrm{kg}]$,

MET - metabolic equivalent of task. 
Table 1. The composition of the diets A, B and C, the mass and caloric content of each constituent [7, 27].

\begin{tabular}{|l|c|c|}
\hline \multicolumn{1}{|c|}{ Diet composition } & $\begin{array}{c}\text { Edible } \\
\text { weight } \\
{[\mathrm{kg}]}\end{array}$ & $\begin{array}{c}\text { Caloric } \\
\text { content } \\
{[\mathrm{kcal} / 100 \mathrm{~g}]}\end{array}$ \\
\hline Diet A & 0.10 & 41 \\
\hline Carrots: domestic, fresh, raw & 0.10 & 62 \\
\hline Whole wheat: domestic, cooked & 0.25 & 141 \\
\hline Soybeans: overseas by boat, cooked & 0.10 & 52 \\
\hline Apples: domestic, fresh, raw & 0.10 & 35 \\
\hline Diet B & 0.30 & 166 \\
\hline Green beans: EU, boiled & 0.10 & 273 \\
\hline Potatoes: cooked & 0.10 & 47 \\
\hline Pork meat: cooked & & \\
\hline Orange: overseas by boat & 0.10 & 60 \\
\hline Diet C & 0.20 & 130 \\
\hline Vegetables: frozen, cooked & 0.10 & 206 \\
\hline Rice: overseas by boat, cooked & 0.10 & 86 \\
\hline Beef: domestic, fresh, cooked & \\
\hline Tropical fruits: by plane, raw & & \\
\hline
\end{tabular}

Meal A has a total of $1.07 \mathrm{kWh} / \mathrm{kg}$ energy, Meal B $-1.65 \mathrm{kWh} / \mathrm{kg}$ and Meal C $-1.42 \mathrm{kWh} / \mathrm{kg}$, while beef has $2.18 \mathrm{kWh} / \mathrm{kg}$ [7]. For comparison, the energy content of gasoline is $12.22 \mathrm{kWh} / \mathrm{kg}$ and diesel - $12 \mathrm{kWh} / \mathrm{kg}$ [28]. Losses during all stages of the food production, including preparation and consumption, were taken into account.

The caloric contents of the foods are taken from the USDA National Nutrient Database [7]. These values were estimated using the Atwater specific factor system [29], which is a refinement based on a reexamination of the Atwater general factor system introduced in 1896 by W.O. Atwater and his colleagues at the United States Department of Agriculture (USDA). The original system was based on the estimation of the heat of combustion of carbohydrates, proteins, and fats using bomb calorimetry. This was then adjusted for losses in digestion, absorption, and urinary excretion. It yielded a single factor for each substrate: $4 \mathrm{kcal} / \mathrm{g}$ for proteins, $9 \mathrm{kcal} / \mathrm{g}$ for fats and $4 \mathrm{kcal} / \mathrm{g}$ for carbohydrates. This method has been widely used due to its apparent simplicity. The refined Atwater specific factor system introduced by Merrill and Watt in 1955 [29] instead of using average factors, derived different factors for proteins, fats, and carbohydrates, depending on the foods in which they are found. Both methods are based on Metabolizable Energy (ME), which is defined as follows:

$$
M E=G E-(F E+G a E+U E+S E)
$$

where GE - Gross energy of food,

FE - Faecal energy,

GaE - Combustible gas (from fermentation), UE - Urinary energy, SE - Surface energy.

The complete human body energy balance is presented in the schematics given in Appendix D [30].

It should be noted that the Atwater system has been criticized in the literature for its inaccuracy in predicting the caloric content of certain foods [31], however, no real alternatives have been proposed. There are few other systems in the literature, such as Net Metabolizable Energy System which is based on NME rather than ME. NME values resulted from further accounting for heat generated due to microbial fermentation, as well as obligatory thermogenesis, which is the

Page 4 of 15 energy that cannot be used for the production of the Adenosine Triphosphate (ATP), i.e. the molecule known for storing and transporting chemical energy. However, in the current study, the Atwater specific factor system is used as the most standardized and widely used system available for the time being, while still offering a reasonable accuracy.

\section{$\boldsymbol{U S A}$}

The analysis for the US is done only for a standard US diet. Average caloric intake in the US, including losses, is estimated to be 2534 calories or $2.95 \mathrm{kWh}$ per day. It was obtained from the Loss-Adjusted Food Availability dataset in the USDA Food Availability (Per Capita) Data System [32]. As was described in the "Methodology" section, the GHG emissions of this diet is given in the next section, which then will be divided by the average daily energy consumption to obtain the average amount of $\mathrm{CO} 2 \mathrm{e}$ emitted per kWh of food energy. This can be coupled with the energy expenditure of the transportation methods to arrive at GHG emissions per distance.

\section{Food Production GHGs}

The life-cycle greenhouse gas emissions estimation is quite a challenging task. Methodologies for estimating the carbon footprint have many issues and are still evolving [33]. They are based on Life Cycle Assessment (LCA) guidelines such as ISO 14040 [34] and PAS2050 [35]. These are usually used in conjunction with procedures recommended by the Intergovernmental Panel on Climate Change (IPCC) [36].

$\mathrm{CO} 2$ is not the only gas causing the global warming. Gases like methane and nitrous oxide are also considered to be GHGs. It is a common practice to represent the quantities of other GHGs in the equivalent quantity of $\mathrm{CO} 2$, which is called equivalent carbon dioxide $(\mathrm{CO} 2 \mathrm{e})$. This convention was used in the current study. $\mathrm{CO} 2 \mathrm{e}$ is a quantity of $\mathrm{CO} 2$ that has the same global warming potential (GWP) as a given type and amount of greenhouse gas. GWP is a measure of the ability of a greenhouse gas to cause radiative forcing, or simply how much heat it traps in the atmosphere.

Depending on the country of origin the amount of CO2e emitted while producing a particular type of food can vary substantially. Figure 3 puts the amount of $\mathrm{CO} 2 \mathrm{e}$ released while producing one kilogram of beef in different countries into perspective. 


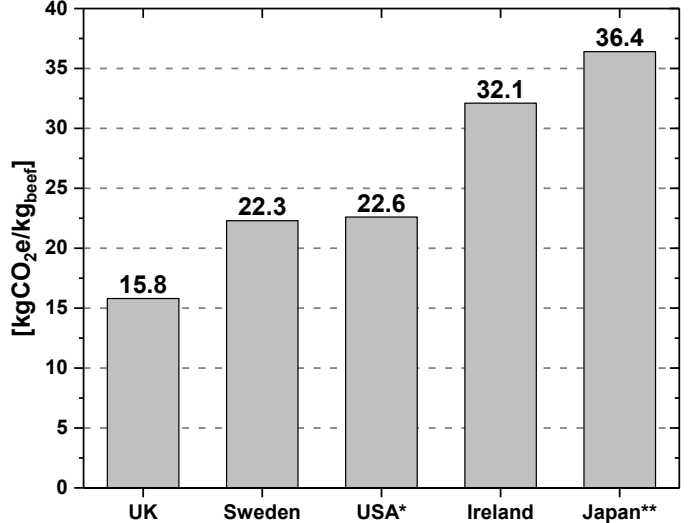

Figure 3. Amount of GHGs emitted per mass of beef produced in different countries [37, 38, 39, 40, 41].

* California Angus beef;

** Kobe beef

Evidently, the values can differ by a factor of two compelling the analysis to be done on a country-by-country basis. The reason behind such dramatic difference lies in many aspects of agricultural and beef production systems of different countries. For example, the fattening of beef cows in Japan requires much higher energy investments than in Sweden (169 MJ/kgbeef [41] vs $25.9 \mathrm{MJ} / \mathrm{kg}_{\text {beef }}$ [38], respectively).

\section{Sweden}

The $\mathrm{CO}_{2} \mathrm{e}$ emissions from the Swedish diets are presented in the Figure 4.

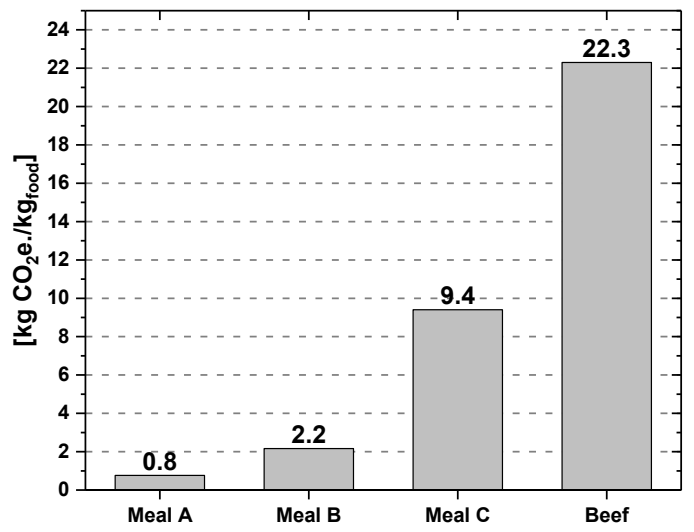

Figure 4. CO2e emissions per kg of different diets in Sweden [27, 38].

Meal A is the most environmentally friendly out of the three contributing only about $0.8 \mathrm{kgCO} 2 \mathrm{e} / \mathrm{kg}_{\text {food, }}$, Meal B contributes almost three times as much, while Meal $\mathrm{C}$ emits almost 12 times more compared to Meal A [27]. Such a big discrepancy can be explained by the fact that some foods are produced domestically, while others are brought from abroad by ships and airplanes. Moreover, environmental burdens of production of different foods can vary drastically.

Page 5 of 15
Heller et al. [42] claims that the standard American diet including all the losses during food production and consumption, emits about 5 $\mathrm{kgCO} 2 \mathrm{e}$ per capita per day, $1.4 \mathrm{kgCO} 2 \mathrm{e}$ of which comes from food wasted in the production and at the retail. Dividing the amount of $\mathrm{CO} 2 \mathrm{e}$ per day by the average energy consumption per day gives the average amount of $\mathrm{CO} 2 \mathrm{e}$ emitted per $\mathrm{kWh}$ of food energy for the standard US diet. This value is approximately equal to 1.7 $\mathrm{kgCO} 2 \mathrm{e} / \mathrm{kWh}$. It is shown later in the article that the environmental impact of this diet lies somewhere between that of the Swedish Meal $\mathrm{B}$ and the British medium-meat diet.

\section{$U K$}

As was mentioned previously, the methodology implemented for the UK differs from that for Sweden or the US. Instead of considering the GHG per kg of food and caloric content of food separately, the amount of $\mathrm{CO}_{2}$ e per energy content of food is directly taken from the paper by Scarborough et al. [43]. This paper reported the GHG emissions for a number of diet types, out of which three most popular were selected: vegetarian ( 0 gmeat/day), medium meat (50-99 gmeat/day), and high meat ( $\geq 100$ gmeat/day) diet. The values for beefeaters are obtained in the same way as in the analysis for Sweden, with $\mathrm{gCO}_{2}$ e per gbeef taken from the paper by Williams et al [37]. In his study, Scarborough estimated the energy content of food using a modified Atwater system. The values of the GHG emissions per diet type are summarized in Figure 5.

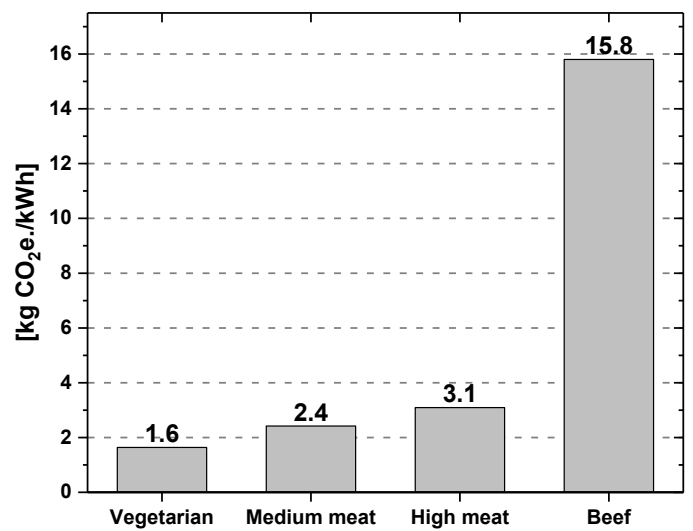

Figure 5. CO2e emissions per energy content of different diets in the UK [37, 43].

Again, beef accounted for the highest GHG emissions, and the environmental impact of each diet was decreasing with the reduction in the amount of meat in the diet.

\section{Results}

The results are presented on a country-by-country basis, first, for one and five persons separately, then for varying number of persons commuting. The values are grouped according to diet type and transportation method. For purposes of comparison, the emissions of the vehicles in all three countries are based on the NEDC. 


\section{Sweden}

Figure 6 shows that, if a person decides to eat meat to replace the calories lost commuting, the amount of GHGs that this person emits driving a conventional ICE vehicle are two to five times lower than those emitted walking, running or bicycling. This difference is even bigger when comparing to the more efficient Toyota Prius (HEV). If five people take a car (Figure 7), the amount of GHGs emitted is up to 22 times less than if they had chosen the other means of transportation and compensated for lost calories by eating meat.

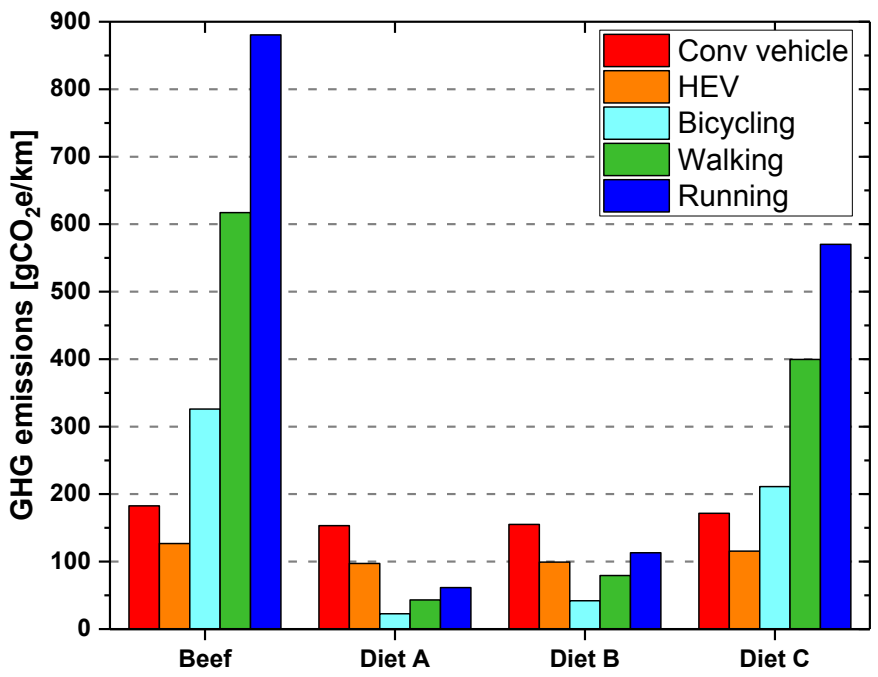

Figure 6. CO2e emissions of one person per km by diet type and transportation method in Sweden.

When more balanced diets are considered, the results become ambiguous. First, comparing environmentally friendly diet A to either conventional vehicle or HEV, it is apparent that, irrespective of whether a person was bicycling, walking or running, the $\mathrm{CO} 2 \mathrm{e}$ emissions of cars are two to seven times higher. However, a more popular diet B has emission levels comparable to that of cars. Even though it is considerably lower than that for ICE cars, it turns out to be quite close to the emissions of HEV: bicycling and walking being $60 \%$ and $20 \%$ lower, respectively, while running being $14 \%$ higher. When five people commute, as seen from Figure 7, even the most environmentally friendly diet A emits same or higher amount of $\mathrm{CO} 2 \mathrm{e}$ as cars, while diet $\mathrm{C}$ emits tremendous $2850 \mathrm{gCO} 2 \mathrm{e} / \mathrm{km}$ for running - 17 times more than driving a hybrid car.

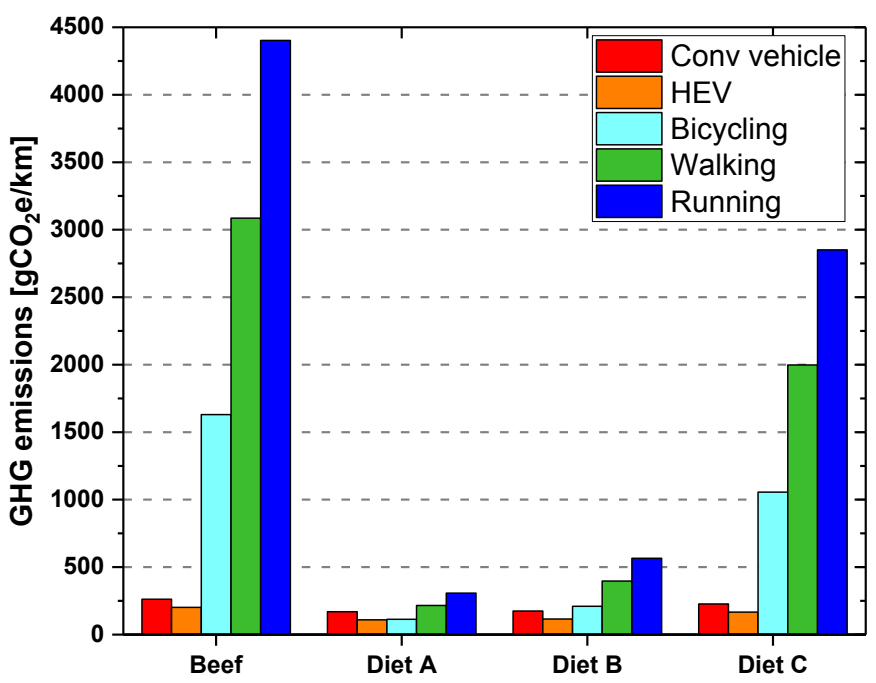

Figure 7. CO2e emissions of five persons per km by diet type and transportation method in Sweden.

Figure 8 presents GHG emissions of different means of transportation as a function of the number of people commuting if they choose the balanced diet B. It can be observed that the borderline, above which hybrid cars like Prius start having lower $\mathrm{CO} 2$ footprint, is 3 persons.

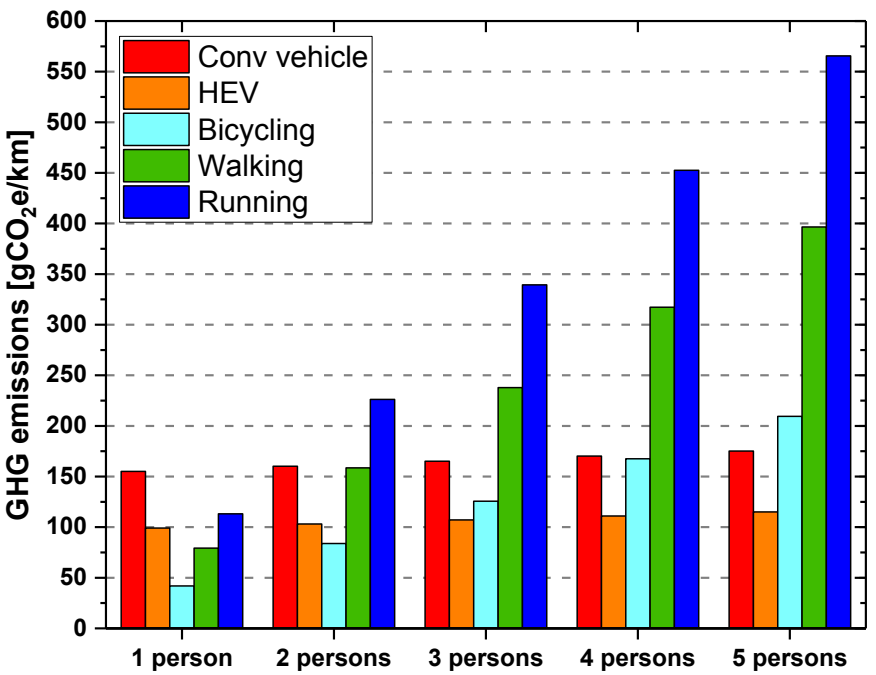

Figure 8. CO2e emissions per km by the number of persons and transportation method in Sweden for diet B.

This borderline for less efficient conventional cars is 4 persons. Bicycling is the most eco-friendly option, being quite far below walking and running. If bicycling is excluded from the comparison, the borderline where cars become more eco-friendly is 2 persons traveling.

If a person is less eco-conscious, meaning one does not pay much attention to the origins of the food he/she consumes, the carbon footprint of such a person might easily end up close or even above the values reported for diet $\mathrm{C}$ (Figure 9). As shown previously, even if there is only one person in the car the amount of GHGs emitted by this person and a car is less than that from one person bicycling. Five people bicycling, on the other hand, are emitting $1056 \mathrm{CO} 2 \mathrm{e} / \mathrm{km}$, which is 4.6 and 6.3 times more than a conventional car and a hybrid, respectively. The effect of diet $\mathrm{C}$ was presented in more details in Figure 9 as a function of the number of people commuting. 


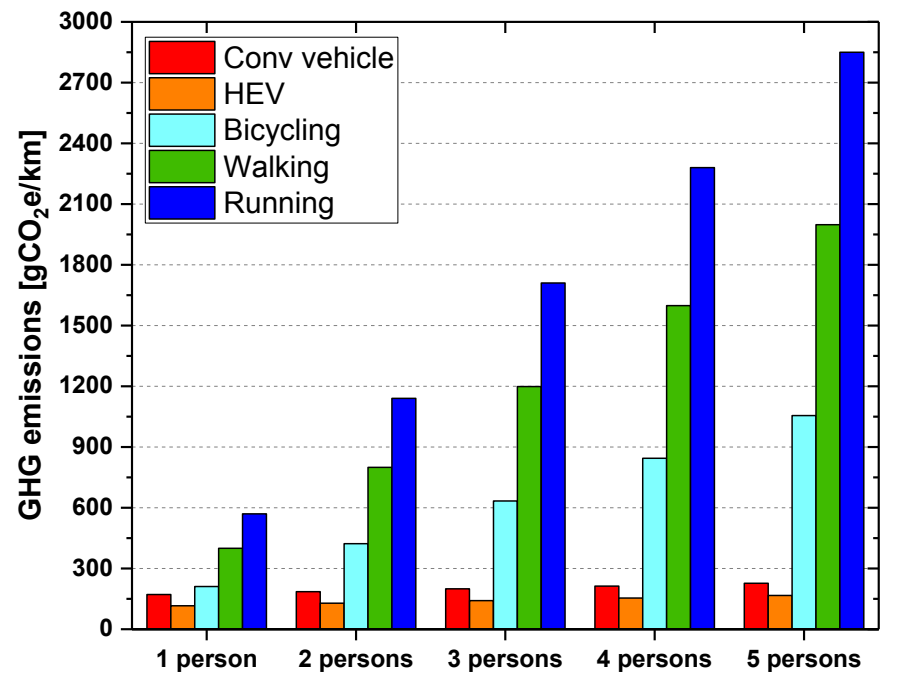

Figure 9. CO2e emissions per $\mathrm{km}$ by the number of persons and transportation method in Sweden for diet C.

It is also interesting to break down the emissions of a car with five people inside by the sources. The importance of the choice of food can easily be appreciated based on the difference between emission distributions in Figures 10 and 11 . If the driver and the passengers ate steak, together they account for almost $40 \%$ of the total emissions. However, if they choose the environmentally friendly diet $\mathrm{A}$, this fraction is only $4 \%$. Another factor to be considered is the mass of the people in the car. The increase in the fuel consumption of the car owing to added mass results in $7-8 \%$ higher $\mathrm{CO} 2 \mathrm{e}$ emissions.

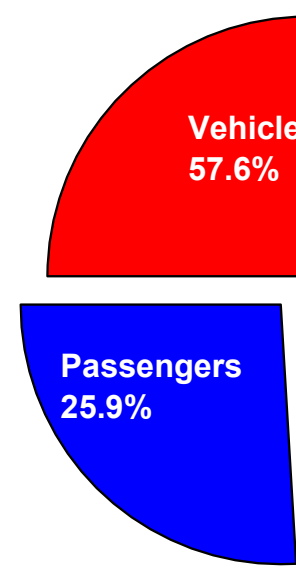

\section{Vehicle baseline} $57.6 \%$

Figure 10. Emissions distribution for five persons riding a car in Sweden if they choose beef diet.

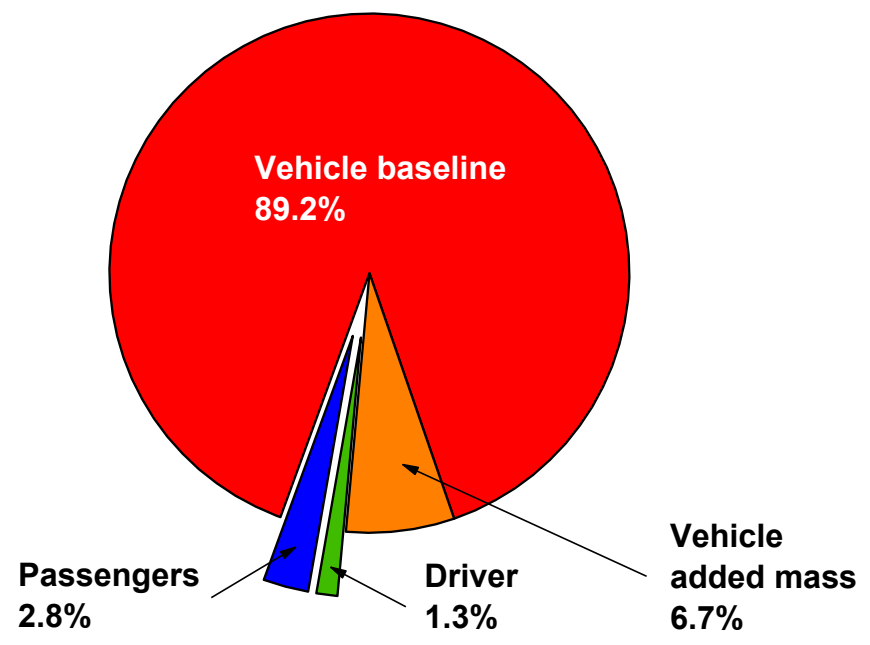

Figure 11. Emissions distribution for five persons riding a car in Sweden if they choose the environmentally friendly $\operatorname{diet} \mathbf{A}$.

\section{UK}

The beef eaters in the UK, once again, cause the biggest impact on the environment when they run, accounting for almost 624 $\mathrm{gCO}_{2} \mathrm{e} / \mathrm{km}$, which is 3.6 times more than a conventional car and over 5 times more than HEV, as seen from Figure 12. Bicycling, as previously, while being the least harmful of the physical activities considered, still has significantly higher emissions than both the ICE vehicle and the hybrid.

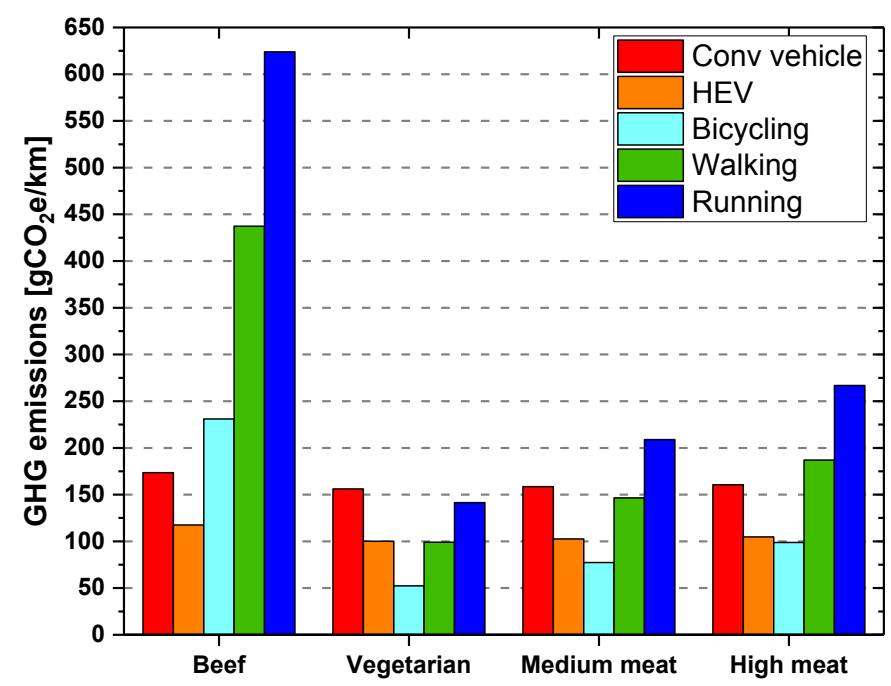

Figure 12. CO2e emissions per km by diet type and transportation method in the UK for one person commuting.

Among the more normal diets the most eco-friendly one is the vegetarian diet. It causes similar GHG emission for walking and driving a hybrid car, as well as for running and driving a conventional car (the latter are only 9\% apart). Bicycling, however, is the cleanest transportation method and accounts for only $52 \mathrm{gCO} 2 \mathrm{e} / \mathrm{km}$. Medium-meat diet has approximately $50 \%$ greater impact than vegetarian, resulting in nearly same emissions for walking and driving a normal car. Running has a significantly higher impact while bicycling has lower. 
High-meat diet ( $\geq 100$ gmeat/day) has even higher $\mathrm{CO}_{2}$ footprint, resulting in $99 \mathrm{gCO}_{2} \mathrm{e} / \mathrm{km}$ even for the most environmentally friendly method of transport that is bicycling. This is very close to the emissions from HEV, though, considerably lower than those of an average car. Walking and running result in up to 2.5 times more emissions than cars.

The results of analysis of five people commuting are unambiguous. Figure 13 demonstrates that the emissions of five people walking, running and even bicycling are drastically higher than those of both conventional vehicles and hybrids.

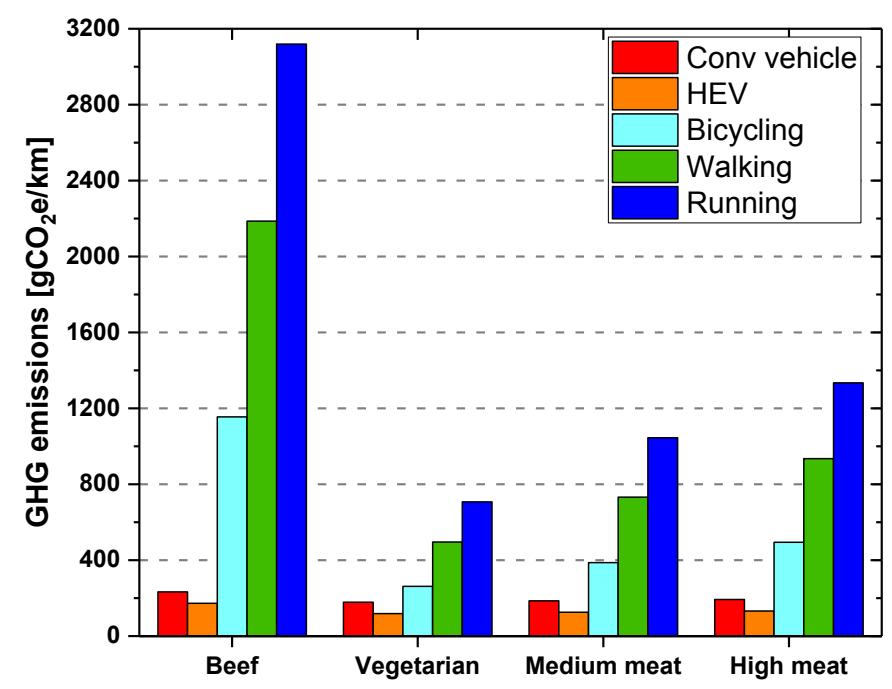

Figure 13. $\mathrm{CO} 2 \mathrm{e}$ emissions per km by diet type and transportation method in the UK for five persons commuting.

Figure 14 presents the GHG emissions associated with medium-meat diet as a function of the number of people commuting. It can be seen that two or more people commuting in the same car emit less $\mathrm{CO} 2 \mathrm{e}$ than two or more people walking or running. If the people prefer bicycling, however, the borderline shifts to three people traveling. Furthermore, the borderline for the UK is one person below than that for Sweden, which is mostly due to higher GHG emissions associated with non-meat products in the UK. This comes from the fact that the meat production in the UK has lower $\mathrm{CO} 2$ footprint than in Sweden, as was shown previously in the paper.

As an extreme case, five people running were found to be emitting $1045 \mathrm{gCO} 2 \mathrm{e} / \mathrm{km}$, about 8 times the emissions of the people riding Toyota Prius (HEV).

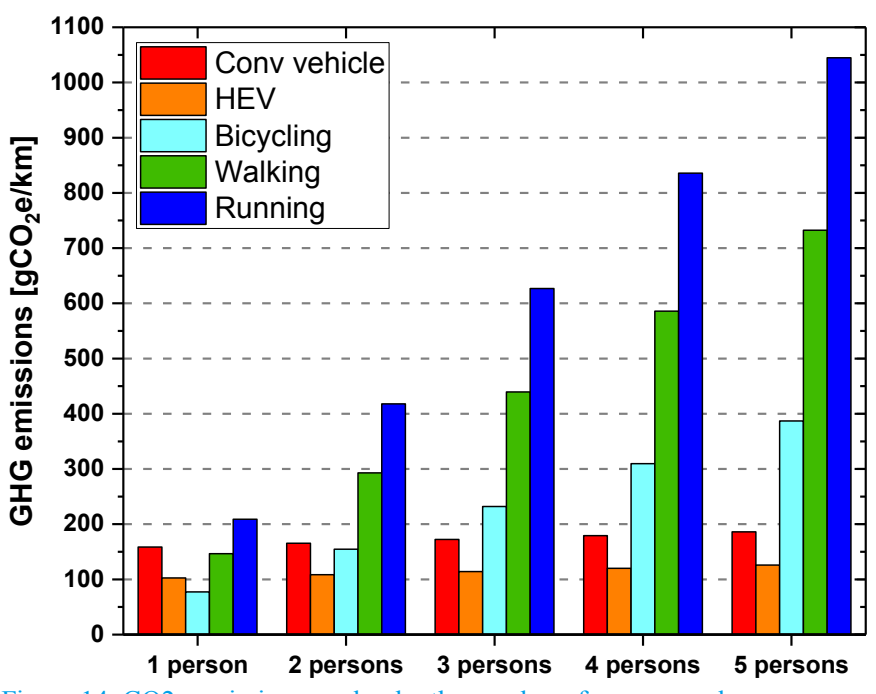

Figure 14. CO2e emissions per $\mathrm{km}$ by the number of persons and transportation method in the UK for the medium-meat diet.

\section{$\boldsymbol{U S A}$}

As was mentioned previously, the analysis for the US differs from that for Sweden and the UK. The fundamental difference is that a standard diet is chosen based on the statistical analysis of the US market, meaning the use of basic food commodities was estimated by tracking their depletion in the marketplace. As a result, instead of calculating emissions for several different diets, one common diet prevailing among the US citizens was chosen. The resulting emissions are visualized in Figure 15. It can be concluded that the standard US diet's $\mathrm{CO}_{2}$ intensity can be positioned in between the Swedish diet B and the British medium-meat diet. It should be noted that the dark chequered segments on top of each bar of the graph indicate the losses associated with food production and consumption in the US and are given as a complementary insight into how much more GHGs are emitted because of food waste. It causes roughly $30 \%$ of the total emissions in the case of walking, bicycling and running, but is much less significant in case of driving a car - only 1$4 \%$. The figure should be compared to the previous figures with the chequered areas included, since the $\mathrm{CO}_{2}$ analysis in the context of other countries encompassed such losses.

The results indicate that the GHG emissions attributed to one person walking in the US are almost the same as driving a hybrid vehicle like Toyota Prius, and approximately equal $103 \mathrm{gCO} 2 \mathrm{e} / \mathrm{km}$. Running emits $43 \%$ more $\mathrm{CO} 2$, i.e., $147 \mathrm{gCO} 2 \mathrm{e} / \mathrm{km}$, which is very close to the emissions of a conventional vehicle. Bicycling is still significantly cleaner, at $54 \mathrm{gCO} 2 \mathrm{e} / \mathrm{km}$. The borderline above which cars become a better option in terms of emissions is three to four persons commuting, if bicycling is included in the comparison, and drops down to two persons if bicycling is not an option. 


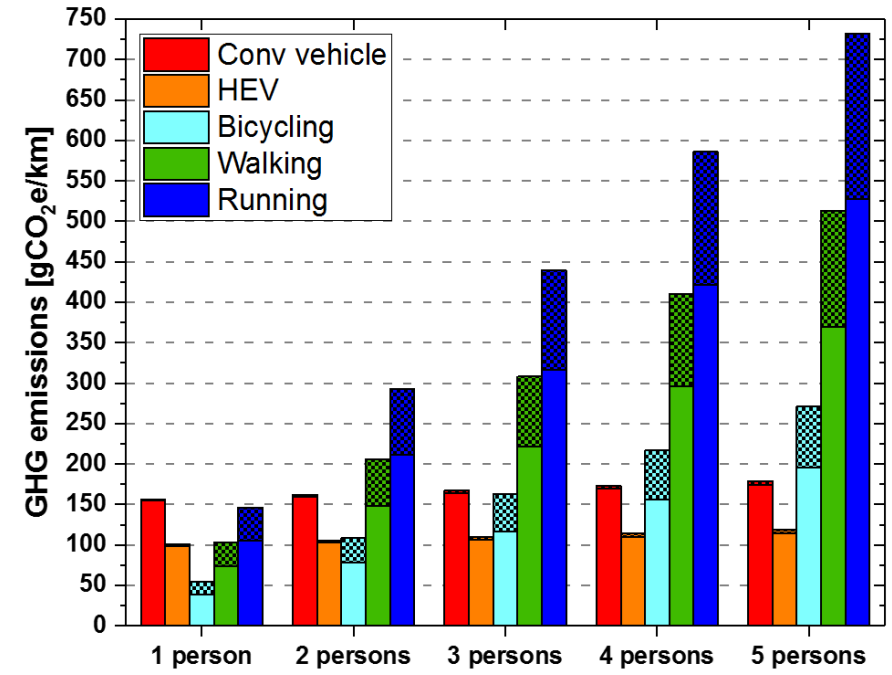

Figure 15. $\mathrm{CO} 2 \mathrm{e}$ emissions per $\mathrm{km}$ with the standard diet as a function of the number of persons and transportation method in the US. The dark chequered segments on top of each bar indicate emissions attributed to the losses in production and consumption of food.

\section{Summary/Conclusions}

This study compares the amount of greenhouse gas emitted per distance traveled walking, running, bicycling, and driving a car. The well-to-wheels analysis is employed to estimate emissions of vehicles. The active modes of transport are evaluated on the basis of the life cycle assessment of food and the estimation of the energy expenditure associated with the aforementioned activities. The conclusions of the study are summarized as follows:

1. If a person has a diet high in meat products $(>100 \mathrm{~g}$ of beef per meal), and/or the diet contains many products imported from abroad, it is generally better in terms of GHG emissions to take a car than walking, bicycling or running.

2. For people choosing only foods with low carbon footprint, as well as vegetarians, it is better to walk or take a bicycle than driving a car. Running, depending on circumstances, can be either better or worse than driving.

3. If three or more people commute together, it is generally better in terms of GHG emissions to take a car (the threshold may vary from two to four depending on the country).

4. A person having a diet with a medium amount of meat emits approximately the same $\mathrm{CO}_{2} \mathrm{e} / \mathrm{km}$ walking, as driving a hybrid car. Depending on the country this value can be either higher or lower. However, if the car has a conventional powertrain, it is better for the environment if the person walks or rides a bicycle. For running it is the opposite.

5. Out of all the human-powered modes of transportation the most environmentally friendly one is bicycling, while the least eco-friendly is running.
6. About $40 \%$ of the vehicle $\mathrm{CO}_{2}$ emissions come from the driver and the passengers if there are five beef-eaters in the car. If the people are very environmentally conscious, this percentage can reduce down to $4 \%$.

7. Approximately $20 \%$ of the GHG emissions of a person are due to respiration. However, these emissions should not be considered climate changing as they are a part of the natural carbon cycle.

\section{References}

1. Maibach, Edward, Linda Steg, and Jillian Anable. "Promoting physical activity and reducing climate change: Opportunities to replace short car trips with active transportation." Preventive medicine 49, no. 4 (2009): 326-327.

2. Haines, Andy, Anthony J. McMichael, Kirk R. Smith, Ian Roberts, James Woodcock, Anil Markandya, Ben G. Armstrong et al. "Public health benefits of strategies to reduce greenhousegas emissions: overview and implications for policy makers." The Lancet 374, no. 9707 (2009): 2104-2114.

3. Woodcock, James, and Rachel Aldred. "Cars, corporations, and commodities: Consequences for the social determinants of health." Emerging Themes in Epidemiology 5, no. 1 (2008): 4.

4. Rabl, Ari, and Audrey De Nazelle. "Benefits of shift from car to active transport." Transport policy 19, no. 1 (2012): 121-131.

5. Komanoff, Charles, Cora Roelofs, Jon Orcutt, and Brian Ketcham. "Environmental benefits of bicycling and walking in the United States." Transportation Research Record 1405 (1993).

6. Stanley, John K., David A. Hensher, and Chris Loader. "Road transport and climate change: Stepping off the greenhouse gas." Transportation Research Part A: Policy and Practice 45, no. 10 (2011): 1020-1030.

7. "National nutrient database for standard reference release 28," United States Department of Agriculture Agricultural Research Service, Tech. Rep., 2015.

8. BARLOW, Tim J., S. Latham, I. S. McCrae, and P. G. Boulter. "A reference book of driving cycles for use in the measurement of road vehicle emissions." TRL Published Project Report (2009).

9. https://www.opel.ie/tools/wltp-drivingcyclefuelconsumption.html/.

10. https://www.toyota-europe.com/new-cars/prius/index/specs.

11. Edwards, Robert, Vincent Mahieu, Jean-Claude Griesemann, Jean-François Larivé, and David J. Rickeard. Well-to-wheels analysis of future automotive fuels and powertrains in the European context. No. 2004-01-1924. SAE Technical Paper, 2004.

12. Edwards, Robert, Jean-Francois Larivé, David Rickeard, and Werner Weindorf. "Well-to-Tank Report Version 4. a." JC Well-to-Wheels Analysis, Joint Research Centre, Luxembourg (2014).

13. Pagerit, Sylvain, P. Sharer, and A. Rousseau. Fuel economy sensitivity to vehicle mass for advanced vehicle powertrains. No. 2006-01-0665. SAE Technical Paper, 2006.

14. Passmore, R., and Jm VGA Durnin. "Human energy expenditure." Physiological reviews 35, no. 4 (1955): 801-840.

15. Hall, Cameron, Arturo Figueroa, B. O. Fernhall, and Jill A. Kanaley. "Energy expenditure of walking and running: 
comparison with prediction equations." Medicine and science in sports and exercise 36 (2004): 2128-2134.

16. "Estimating energy expenditure," University of Colorado Hospital, 2004.

17. Léger, L., and D. Mercier. "Gross energy cost of horizontal treadmill and track running." Sports medicine 1, no. 4 (1984): 270-277.

18. Jette, M., K. Sidney, and G. Blümchen. "Metabolic equivalents (METS) in exercise testing, exercise prescription, and evaluation of functional capacity." Clinical cardiology 13, no. 8 (1990): 555-565.

19. Cohen, M., and M. Heberger. "Driving vs. walking: Cows, climate change, and choice." Pacific Institute (2008).

20. Edholm, O. G., J. G. Fletcher, Elsie M. Widdowson, and R. A. McCance. "The energy expenditure and food intake of individual men." British Journal of Nutrition 9, no. 3 (1955): 286-300.

21. Levine, James A., Sara J. Schleusner, and Michael D. Jensen. "Energy expenditure of nonexercise activity-." The American journal of clinical nutrition 72, no. 6 (2000): 1451-1454.

22. Dong, Linda, Gladys Block, and Shelly Mandel. "Activities contributing to total energy expenditure in the United States: results from the NHAPS Study." International Journal of Behavioral Nutrition and Physical Activity 1, no. 1 (2004): 4.

23. Chang, Young-Hui, and Rodger Kram. "Metabolic cost of generating horizontal forces during human running." Journal of Applied Physiology 86, no. 5 (1999): 1657-1662.

24. Farley, Claire T., and THOMAS A. McMAHON. "Energetics of walking and running: insights from simulated reduced-gravity experiments." Journal of Applied Physiology 73, no. 6 (1992): 2709-2712.

25. Kram, Rodger, and C. Richard Taylor. "Energetics of running: a new perspective." Nature 346, no. 6281 (1990): 265.

26. Roberts, Thomas J., R. O. D. G. E. R. Kram, PETER G. Weyand, and C. Richard Taylor. "Energetics of bipedal running. I. Metabolic cost of generating force." Journal of Experimental Biology 201, no. 19 (1998): 2745-2751.

27. Carlsson-Kanyama, Annika, and Alejandro D. González. "Potential contributions of food consumption patterns to climate change-." The American journal of clinical nutrition 89, no. 5 (2009): 1704S-1709S.

28. Johansson, Bengt. Combustion Engines. Department of Energy Sciences, Lund University, 2014.

29. Merrill, A. L., and B. K. Watt. "Energy value of foods: basis and derivation. Washington, DC: United States Department of Agriculture, 1973." Agriculture handbook 74.

30. Maclean, William, James Harnly, Junshi Chen, Simon Chevassus-Agnes, G. Gilani, Geoffrey Livesey, and P. Warwick. "Food energy-Methods of analysis and conversion factors." In Food and Agriculture Organization of the United Nations Technical Workshop Report, vol. 77. 2003.

31. Novotny, Janet A., Sarah K. Gebauer, and David J. Baer. "Discrepancy between the Atwater factor predicted and empirically measured energy values of almonds in human diets." The American journal of clinical nutrition 96, no. 2 (2012): 296-301.

32. "https://www.ers.usda.gov/data-products/food-availability-percapita-data-system/."

33. Pandey, Divya, Madhoolika Agrawal, and Jai Shanker Pandey. "Carbon footprint: current methods of estimation." Environmental monitoring and assessment 178, no. 1-4 (2011): 135-160.

34. Guinée, Jeroen B. "Handbook on life cycle assessment operational guide to the ISO standards." The international journal of life cycle assessment 7, no. 5 (2002): 311.

Page 10 of 15
35. Specification, Publicly Available. "Specification for the assessment of the life cycle greenhouse gas emissions of goods and services." BSI British Standards. ISBN 978, no. 0 (2008): 580 .

36. Eggleston, Simon, Leandro Buendia, and Kyoko Miwa. 2006 IPCC guidelines for national greenhouse gas inventories [recurso electrónico]: waste. Kanagawa, JP: Institute for Global Environmental Strategies, 2006.

37. Williams, A., E. Audsley, and D. Sandars. "Determining the environmental burdens and resource use in the production of agricultural and horticultural commodities: Defra project report IS0205." Zu finden in: http://randd. defra. gov. uk/Default. aspx(2006).

38. Cederberg, Christel, and Magnus Stadig. "System expansion and allocation in life cycle assessment of milk and beef production." The International Journal of Life Cycle Assessment 8, no. 6 (2003): 350-356.

39. Casey, J. W., and N. M. Holden. "Greenhouse gas emissions from conventional, agri-environmental scheme, and organic Irish suckler-beef units." Journal of Environmental Quality 35, no. 1 (2006): 231-239.

40. Stackhouse-Lawson, K. R., C. A. Rotz, J. W. Oltjen, and F. M. Mitloehner. "Carbon footprint and ammonia emissions of California beef production systems." Journal of Animal Science 90, no. 12 (2012): 4641-4655.

41. Ogino, Akifumi, Hideki Orito, Kazuhiro Shimada, and Hiroyuki Hirooka. "Evaluating environmental impacts of the Japanese beef cow-calf system by the life cycle assessment method." Animal Science Journal 78, no. 4 (2007): 424-432.

42. Heller, Martin C., and Gregory A. Keoleian. "Greenhouse gas emission estimates of US dietary choices and food loss." Journal of Industrial Ecology 19, no. 3 (2015): 391-401.

43. Scarborough, Peter, Paul N. Appleby, Anja Mizdrak, Adam DM Briggs, Ruth C. Travis, Kathryn E. Bradbury, and Timothy J. Key. "Dietary greenhouse gas emissions of meat-eaters, fisheaters, vegetarians and vegans in the UK." Climatic change125, no. 2 (2014): 179-192.

44. Casaburi, Richard, THOMAS J. Barstow, T. E. R. R. Y. Robinson, and K. A. R. L. M. A. N. Wasserman. "Influence of work rate on ventilatory and gas exchange kinetics." Journal of Applied Physiology 67, no. 2 (1989): 547-555.

45. Van der Walt, W. H., and C. H. Wyndham. "An equation for prediction of energy expenditure of walking and running." Journal of Applied Physiology 34, no. 5 (1973): 559563.

46. A. C. of Sports Medicine, Guidelines for exercise testing and prescription. Williams \& Wilkins, 1991.

47. Ejaimi, Gamal, and Sittelnissa Saeed. "An Introduction to Airway Assessment and Management (Concise Airway Anatomy and Pathophysiology)."

48. Glenny, Robb W., and H. Thomas Robertson. Introduction to cardiopulmonary exercise testing. New York: Springer, 2013.

\section{Contact Information}

Rafig Babayev

Clean Combustion Research Center (CCRC)

King Abdullah University of Science and Technology (KAUST) Building 5, Level 4, 4204-WS10, Thuwal 23955-6900, Saudi Arabia rafig.babayev@kaust.edu.sa rafik.babayev95@gmail.com +966560249272 
Definitions/Abbreviations

$\begin{array}{ll}\text { GHG } & \text { Greenhouse Gas } \\ \text { ICE } & \text { Internal Combustion Engine } \\ \text { HEV } & \text { Hybrid Electric Vehicle } \\ \text { BEV } & \text { Battery Electric Vehicle } \\ \text { LCA } & \text { Life Cycle Assessment } \\ \text { CO2e } & \text { equivalent carbon dioxide }\end{array}$

WTW

WTT

TTW

NEDC

vó $\dot{2}_{2}$ peak

MET

ME

ATP
Well-to-Wheels

Well-to-Tank

Tank-to-Wheels

New European Driving Cycle

peak aerobic power

Metabolic Equivalent of Task

Metabolizable Energy

Adenosine Triphosphate

Page 11 of 15 


\section{Appendix}

\section{Appendix $A$}

\section{Well-to-Wheels Analysis}

$$
\begin{aligned}
F C\left[\frac{g_{f}}{k m}\right] & =\frac{F C\left[\frac{L}{100 \mathrm{~km}}\right]}{100} \times \rho_{f}\left[\frac{g_{f}}{L}\right] \\
T T W G H G\left[\frac{g C O_{2}}{k m}\right] & =T T W G H G\left[\frac{g C O_{2}}{g_{f}}\right] \times F C\left[\frac{g_{f}}{k m}\right] \\
W T T G H G\left[\frac{g C O_{2} e}{g_{f}}\right] & =W T T G H G\left[\frac{g C O_{2} e}{M J_{f}}\right] \times L H V\left[\frac{M J_{f}}{g_{f}}\right] \\
W T T G H G\left[\frac{g \mathrm{CO}_{2} e}{k m}\right] & =W T T G H G\left[\frac{g C O_{2} e}{g_{f}}\right] \times F C\left[\frac{g_{f}}{k m}\right] \\
W T W G H G\left[\frac{g C O_{2} e}{k m}\right]= & T T W G H G\left[\frac{g C O_{2} e}{k m}\right]+W T T G H G\left[\frac{g \mathrm{CO}_{2} e}{k m}\right]
\end{aligned}
$$

Where: $F C\left[\frac{L}{100 \mathrm{~km}}\right]-$ NEDC fuel consumption of a vehicle [28],

TTW GHG $\left[\frac{g \mathrm{gO}_{2}}{g_{f}}\right]$ - Amount of $\mathrm{CO}_{2}$ emitted per amount of fuel burned estimated based on the stoichiometric gasoline combustion reaction,

WTT GHG $\left[\frac{g \mathrm{gO}_{2} e}{M J_{f}}\right]-$ Well-to-tank GHGs emitted on a fuel energy basis [12],

\section{Appendix B}

\section{Respiratory Carbon Dioxide Emissions}

Human respiratory system obtains oxygen for use by body cells and expels carbon dioxide produced by the cells. The primary respiration reaction occurs between glucose and oxygen producing water, ATP and carbon dioxide. According to Jette et al. walking, bicycling and running can be considered light-, moderate-, and very heavy-intensity physical activities, respectively [18]. The workload associated with these activities were summarized in the second column of Table 2 .

A study done by Richard Casaburi and his colleagues at Harbor-UCLA Medical Center explored the influence of workload on human gas exchange characteristics and suggests the human volumetric carbon dioxide output for the aforementioned activities listed in the third column of Table 2 [44]. Assuming the speed of walking, bicycling and running to be $4.8 \mathrm{~km} / \mathrm{h}, 16 \mathrm{~km} / \mathrm{h}$ and $9.7 \mathrm{~km} / \mathrm{h}$, respectively, and taking the density of $\mathrm{CO}_{2}$ as 1842 $\mathrm{g} / \mathrm{m}^{3}$, the average amount of $\mathrm{CO}_{2}$ per unit distance was estimated and given in the fourth column of Table 2 .

Table 2. Workload and carbon dioxide emissions attributed to human breathing [18, 44].

\begin{tabular}{|l|l|l|l|}
\hline \multirow{2}{*}{} & Workload & \multicolumn{2}{|c|}{$\mathrm{CO}_{2}$ emitted } \\
\cline { 2 - 4 } & \multicolumn{1}{|c}{$[\mathrm{L}]$} & \multicolumn{1}{|c|}{$[\mathrm{min}]$} & {$\left[\mathrm{gCO}_{2} / \mathrm{km}\right]$} \\
\hline Walking & $28-69$ & 1 & 22.9 \\
\hline Bicycling & $70-104$ & 1.5 & 10.4 \\
\hline Running & $140-174$ & 2.5 & 28.6 \\
\hline
\end{tabular}

It can be concluded that the respiratory $\mathrm{CO} 2$ emissions add approximately $20 \%$ to the total GHG emissions of an average person in the US, regardless of whether the person was walking, bicycling or running. In Sweden, this ratio is a little higher at 25-29\%, for Diet B. In the UK, however, it is a little lower at $10-14 \%$, for the medium-meat diet.

Page 12 of 15 
Knowing the concentration of $\mathrm{CO} 2$ in the exhaled air, the "equivalence ratio" of a human body can also be estimated. The calculations are presented in Appendix E.

\section{Appendix C}

\section{Peak Aerobic Power Calculations}

\section{Running}

1. Leger et al. [17]:

$$
V \dot{O}_{2} \text { peak }\left[\frac{\mathrm{ml}}{\mathrm{kg} \times \min }\right]=2.209+3.1633 v
$$

2. Van der Walt et al. [45]:

$$
V \dot{O}_{2} \text { peak }\left[\frac{L}{\min }\right]=-0.419+0.03257 m+0.000117 m v^{2}
$$

3. American College of Sports Medicine [46]:

$$
V \dot{O}_{2} \text { peak }\left[\frac{\mathrm{ml}}{\mathrm{kg} \times \min }\right]=0.2\left[\frac{\mathrm{m}}{\mathrm{s}}\right]+0.9\left[\frac{\mathrm{m}}{\mathrm{s}}\right] F+3.5
$$

\section{Walking}

4. Van der Walt et al. [45]:

$$
V \dot{O}_{2} \text { peak }\left[\frac{L}{\min }\right]=0.00599 m+0.000366 m v^{2}
$$

5. American College of Sports Medicine [46]:

$$
V \dot{O}_{2} \text { peak }\left[\frac{\mathrm{ml}}{\mathrm{kg} \times \mathrm{min}}\right]=0.1\left[\frac{\mathrm{m}}{\mathrm{s}}\right]+1.8\left[\frac{\mathrm{m}}{\mathrm{s}}\right] F+3.5
$$

Where: $\quad \mathrm{v}-$ Speed $[\mathrm{km} / \mathrm{h}]$,

$\mathrm{m}$ - Body mass $[\mathrm{kg}]$,

F - Fractional grade. 


\section{Appendix D}

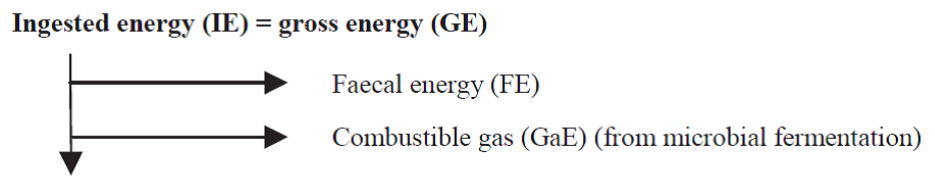

Digestible energy (DE)

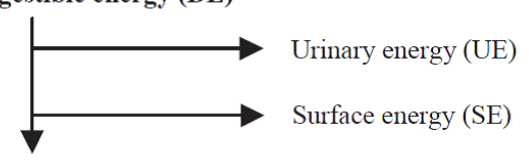

Metabolizable energy (ME)

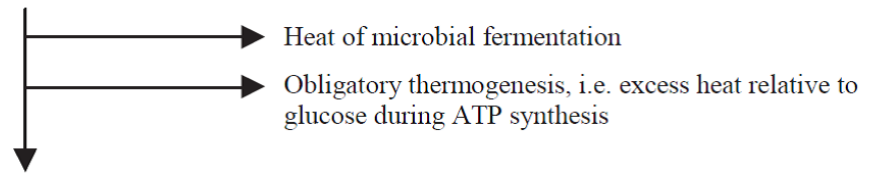

Net (metabolizable) energy (NME)

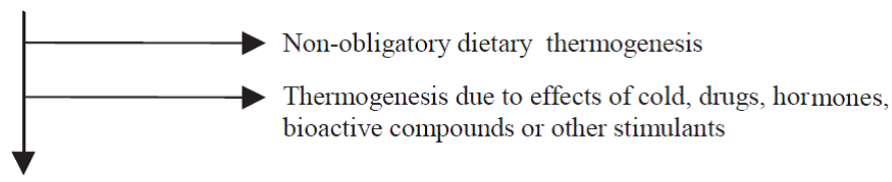

Net energy for maintenance (NE)

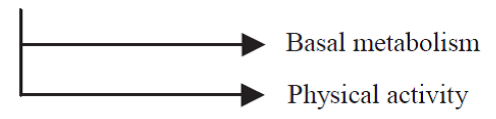

Figure 16. Human body energy balance [30].

\section{Appendix $E$}

\section{Human Equivalence Ratio}

Knowing the $\mathrm{CO} 2$ emissions associated with breathing, the air-fuel equivalence ratio $(\lambda)$ of humans can also be estimated with the following assumptions:

1. Breakdown of glucose is complete

2. Concentration of carbon monoxide is negligible

3. Nitrogen in air does not participate in the reaction.

Then the global chemical equation of cellular respiration becomes:

$C_{a} H_{b} O_{c}+\lambda\left(a+\frac{b}{4}-\frac{c}{2}\right)\left(O_{2}+3.773 N_{2}\right) \rightleftharpoons a C O_{2}+\frac{b}{2} H_{2} O+(\lambda-1)\left(a+\frac{b}{4}-\frac{c}{2}\right) O_{2}+3.773 \lambda\left(a+\frac{b}{4}-\frac{c}{2}\right) N_{2}+A T P$

Where, $\quad a=6, b=12, c=6$.

The wet concentration of carbon dioxide in the exhaled air is approximately 4\% [47]. The Ventilatory Equivalent for Carbon Dioxide (VE/VCO2), which refers to the number of liters of ventilation per liter of CO2 output, is typically 25 and remains at or near this level in healthy individuals with progressive exercise. This indicates that the concentration of carbon dioxide in the expelled air normally does not vary much until the ventilatory threshold is reached [48].

Consequently, defining the concentration of $\mathrm{CO}_{2}$ from equation (E.1) as follows:

$X_{\mathrm{CO}_{2}}=\frac{a}{a+\frac{b}{2}+(\lambda-1)\left(a+\frac{b}{4}-\frac{c}{2}\right)+3.773 \lambda\left(a+\frac{b}{4}-\frac{c}{2}\right)} \quad$ (E.2),

$\lambda$ can be calculated according to:

$\lambda=\frac{a-\left(\frac{b}{4}+\frac{c}{2}\right) X_{\mathrm{CO}_{2}}}{4.773\left(a+\frac{b}{4}-\frac{c}{2}\right) X_{\mathrm{CO}_{2}}}$

Page 14 of 15 
yielding $\lambda \approx 5$.

It should be emphasized that reaction (E.1) does not describe the actual process of the breakdown of glucose. This is rather a summary (global) reaction, since the actual process of cellular respiration involves many steps. However, the chemical kinetics are not important in the context of this study as only the final equilibrium product concentrations are needed for the calculation of $\lambda$. 